\title{
Photometry and spectroscopy of the RS CVn system $\sigma$ Geminorum
}

\author{
X.B. Zhang ${ }^{1,2}$ and R.X. Zhang ${ }^{1}$ \\ 1 Beijing Astronomical Observatory, Chinese Academy of Sciences, Bejing 100101, China \\ e-mail: xzhang@class1.bao.ac.cn \\ 2 Chinese Academy of Sciences-Peking University joint Beijing Astrophysical Center, Beijing 100871, China
}

Received July 22; accepted August 31, 1998

\begin{abstract}
We present new $U B V$ photoelectric photometry and high-resolution $\mathrm{H}_{\alpha}$ spectroscopy of the RS CVn system $\sigma$ Gem made in 1993-1994. A brief photometric analysis for the new light curves is carried out using the Wilson-Devinney method with spot approximation based on the Roche model. The result suggests the probable presence of two cool spots on the K1 III primary around the phases of $\phi=0.6$ and $\phi=0.9$. The spot activity is discussed in connection with the chromospheric $\mathrm{H}_{\alpha}$ emission. The enhanced $\mathrm{H}_{\alpha}$ core emission is found to vary with phase and shows distinct anti-correlation with the spot regions. It may be an indication for the existence of plage regions on $\sigma \mathrm{Gem}$.
\end{abstract}

Key words: stars: $\sigma$ Gem; activity — binaries: close

\section{Introduction}

$\sigma$ Gem $(=$ HR $2973=$ HD 62044$)$ is a well known member of the long-period RS CVn systems. It is a non-eclipsing single-line spectroscopic binary with a long orbital period of 19.605 days (Bopp \& Dempsey 1989). The visible primary is a red giant of spectral type K1 III. Little is known about the secondary component.

The light variability of this star was discovered by Hall et al. (1977). Fried et al. (1983) contributed a detailed photometric study of $\sigma$ Gem and derived a reliable photometric period of 19.423 days, which is shorter than the orbital period. The amplitude of the photometric wave is variable with a maximum value of $0.17 \mathrm{mag}$ in $V$. As a hyperactive star $\sigma$ Gem draws much attention of astronomers. Focusing on the starspot changes over an eight-year period, Strassmeier et al. (1988) analyzed the $V$ light curves and discussed the cyclic variations and spot migration.

Send offprint requests to: X.B. Zhang
With a simplified light curve modeling method they suggested two cool spot regions on the K1 III primary and found a $2.7 \mathrm{yr}$ period of cyclic variation for both spots and a new photometric period of 19.410 days. Later, Olah et al. (1989) investigated the light curve behavior of $\sigma$ Gem from 1986 to 1988 with the same method. They also confirmed large spot changes from year to year and the existence of two active longitudes on $\sigma$ Gem. A detailed study on spot activities of $\sigma$ Gem was recently published by Henry et al. (1995).

Strong CaII $\mathrm{H}$ and $\mathrm{K}$ emission, UV line emission and $\mathrm{X}$-ray emission have also been observed, revealing the unusual manifestations of $\sigma$ Gem with high chromospheric and coronal activity (Schrijver et al. 1995; Elgaray et al. 1997). The $\mathrm{H}_{\alpha}$ core emission has been observed by many authors. First, Smith \& Bopp (1982) noted that the $\mathrm{H}_{\alpha}$ absorption line in $\sigma$ Gem was partly filled in by chromospheric emission. Strassmeier et al. (1986) measured the $\mathrm{H}_{\alpha}$ feature with an average $\mathrm{EW}$ of $972 \mathrm{m \AA}$ and an average $R_{\mathrm{c}}$ of 0.51 . Strassmeier \& Fekel (1990) and Frasca \& Catalano (1994) computed the residual emission separately and got different results. A detailed study of the $\mathrm{H}_{\alpha}$ profile by Eker (1986) showed the line core to vary with the orbital phase in agreement with the location of stellar active regions. Bopp et al. (1988) indicated that the $\mathrm{H}_{\alpha}$ EW varied with rotational phase in 1986 but not obviously in 1987.

From 1993 to 1994, we made almost simultaneous photoelectric $U B V$ and spectroscopic observations. In this paper, we present an analysis of the observations. The spot activity and the chromospheric $\mathrm{H}_{\alpha}$ emission will be discussed in the context of the stellar evolutionary status.

\section{Observations and data reductions}

In the $1993 / 94$ observing season, new $U B V$ photometry of $\sigma$ Gem was carried out at the Xinglong station of Beijing Astronomical Observatory. On 17 nights from 


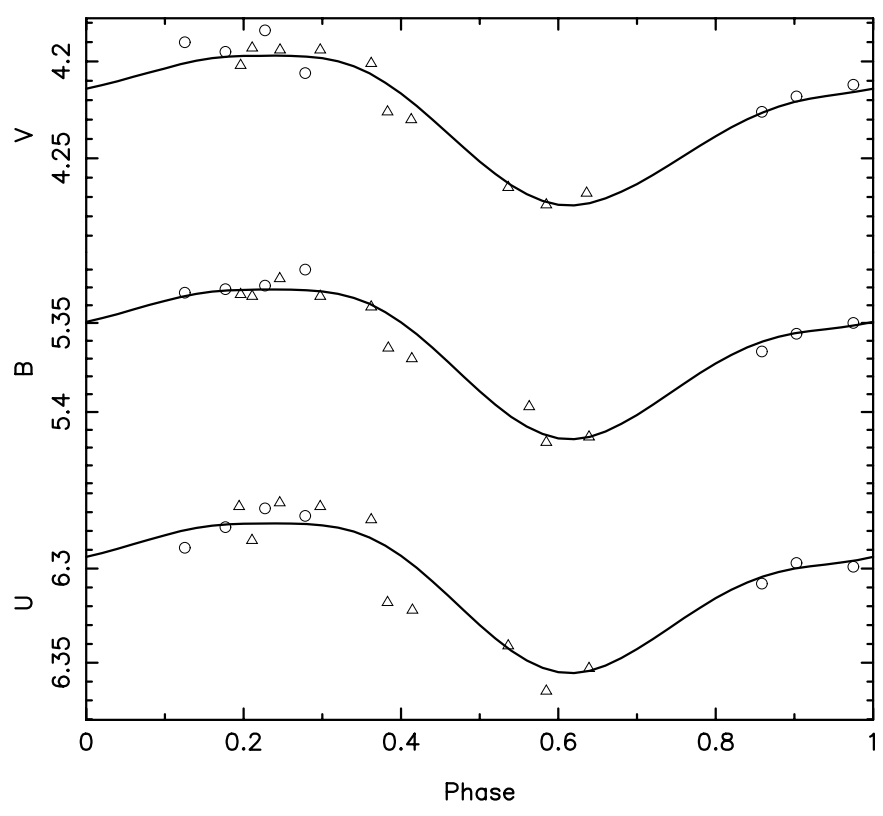

Fig. 1. The new light curves of $\sigma$ Gem observed in 1993/94. Circles denote the measurements obtained in 1993 Jan.-Feb., and the triangles stand for that observed in 1993 Nov.-1994 Jan. Solid lines represent the computed light curves using WD method with two-spot model

1993 January to 1994 January, the observations were made on the $60 \mathrm{~cm}$ reflector using a single channel photometer with a direct amplification receiver. The star $\rho$ Gem (=HD 58946, $V=4.18, U-B=-0.03, B-V=+0.32$, Strassmeier et al. 1988) was adopted as the comparison star. The integration time was $30 \mathrm{~s}$ for both the target and comparison stars in all filters. The accuarcy of the observations was estimated to be about $0.01 \mathrm{mag}$ in all $V, B$ and $U$ bands on most nights. All the measurements were transformed to the $U B V$ standard system and combined into light curves using the photometric ephemeris of Fried et al. (1983),

$\mathrm{JD}($ hel $)=2444686.5+19.423 \times E$.

Table 1 gives the dataset of $U B V$ measurements. We combined the data obtained on one night into one average point because of the long period of the system. The $U B V$ light curves are shown in Fig. 1, where circles and triangles represent the measurements observed in early 1993, and from 1993 November to 1994 January.

Spectroscopic observations were made with the $2.16 \mathrm{~m}$ telescope also at the Xinglong station in 1993 on eight nights from 1993 Jan. 12 to Jan. 19. The spectra were obtained by using the fiber-fed Cassegrain ISIS spectrograph, which was designed specifically for the international MUSICOS observations by Meudon Observatory (Cuby et al. 1992). With a THX $576 \times 384$ CCD detector the ISIS spectrograph yields a resolving power of about $3.610^{4}$ at $\mathrm{H}_{\alpha}$ band. The spectrum covers about $35 \AA$ from 6544.5 to $6579.5 \AA$. The reciprocal dispersion is $2.64 \AA \mathrm{mm}^{-1}$, corresponding to a 2-pixel resolution of
Table 1. Photometric measurements of $\sigma$ Gem

\begin{tabular}{|c|c|c|c|c|c|}
\hline $\begin{array}{l}\mathrm{JD}(\mathrm{Hel}) \\
2440000+\end{array}$ & $\begin{array}{l}V \\
(\mathrm{mag})\end{array}$ & $\begin{array}{l}\mathrm{JD}(\mathrm{Hel}) \\
2440000+\end{array}$ & $\begin{array}{l}B \\
\text { (mag) }\end{array}$ & $\begin{array}{l}\mathrm{JD}(\mathrm{Hel}) \\
2440000\end{array}$ & $\begin{array}{l}U \\
(\mathrm{mag})\end{array}$ \\
\hline 9015.0712 & 4.231 & 9015.0713 & 5.366 & 9015.0714 & 6.312 \\
\hline 9015.0848 & 4.227 & 9015.0830 & 5.366 & 9015.0832 & 6.308 \\
\hline 9015.0925 & 4.222 & 9015.0927 & 5.366 & 9015.0928 & 6.304 \\
\hline 9015.9334 & 4.220 & 9015.9334 & 5.351 & 9015.9335 & 6.300 \\
\hline 9015.9412 & 4.217 & 9015.9413 & 5.360 & 9015.9413 & 6.294 \\
\hline 9017.3190 & 4.211 & 9017.3171 & 5.345 & 9017.3172 & 6.298 \\
\hline 9017.3422 & 4.222 & 9017.3422 & 5.346 & 9017.3423 & 6.293 \\
\hline 9017.3615 & 4.203 & 9017.3630 & 5.357 & 9017.3616 & 6.307 \\
\hline 9020.2452 & 4.193 & 9020.2480 & 5.339 & 9020.2470 & 6.295 \\
\hline 9020.2628 & 4.181 & 9020.2628 & 5.334 & 9020.2610 & 6.287 \\
\hline 9020.2726 & 4.195 & 9020.2726 & 5.327 & 9020.2742 & 6.284 \\
\hline 9021.2612 & 4.194 & 9021.2612 & 5.335 & 9021.2600 & 6.286 \\
\hline 9021.2767 & 4.195 & 9021.2768 & 5.328 & 9021.2768 & 6.270 \\
\hline 9022.2440 & 4.184 & 9022.2440 & 5.329 & 9022.2441 & 6.268 \\
\hline 9023.2365 & 4.206 & 9023.2365 & 5.320 & 9023.2354 & 6.272 \\
\hline 9313.2216 & 4.206 & 9313.2216 & 5.350 & 9313.2216 & 6.299 \\
\hline 9313.2392 & 4.203 & 9313.2393 & 5.344 & 9313.2293 & 6.284 \\
\hline 9313.2586 & 4.193 & 9313.2570 & 5.335 & 9313.2572 & 6.281 \\
\hline 9313.2858 & 4.190 & 9313.2842 & 5.331 & 9313.2844 & 6.278 \\
\hline 9313.3033 & 4.180 & 9313.3030 & 5.317 & 9313.3018 & 6.270 \\
\hline 9313.3208 & 4.184 & 9313.3208 & 5.330 & 9313.3210 & 6.297 \\
\hline 9316.1706 & 4.210 & 9316.1706 & 5.351 & 9316.1706 & 6.276 \\
\hline 9316.1803 & 4.207 & 9316.1803 & 5.342 & 9316.1803 & 6.268 \\
\hline 9316.1992 & 4.204 & 9316.1992 & 5.339 & 9316.2024 & 6.272 \\
\hline 9316.2114 & 4.201 & 9316.2115 & 5.341 & 9316.2106 & 6.290 \\
\hline 9316.2366 & 4.190 & 9316.2350 & 5.336 & 9316.2352 & 6.268 \\
\hline 9316.2463 & 4.193 & 9316.2465 & 5.336 & 9316.2446 & 6.270 \\
\hline 9317.1592 & 4.236 & & & & \\
\hline 9317.1689 & 4.250 & 9317.1690 & 5.372 & & \\
\hline 9317.1903 & 4.224 & & & 9317.2118 & 6.334 \\
\hline 9317.2212 & 4.222 & 9317.2212 & 5.365 & 9317.2212 & 6.323 \\
\hline 9317.2427 & 4.220 & 9317.2442 & 5.375 & 9317.2442 & 6.310 \\
\hline 9336.0350 & 4.226 & 9336.0540 & 5.370 & 9336.0350 & 6.324 \\
\hline 9339.0058 & 4.260 & 9339.0045 & 5.397 & 9339.0046 & 6.327 \\
\hline 9339.0136 & 4.262 & 9339.0124 & 5.398 & 9339.0126 & 6.353 \\
\hline 9339.0214 & 4.272 & & & 9339.0248 & 6.343 \\
\hline 9339.9479 & 4.274 & 9339.9481 & 5.417 & 9339.9471 & 6.374 \\
\hline 9339.9595 & 4.275 & 9339.9596 & 5.417 & 9339.9596 & 6.364 \\
\hline 9340.9806 & 4.269 & 9340.9806 & 5.415 & 9340.9807 & 6.357 \\
\hline 9341.0330 & 4.267 & 9341.0330 & 5.413 & 9341.0330 & 6.348 \\
\hline 9371.2486 & 4.202 & 9371.2486 & 5.337 & 9371.2478 & 6.269 \\
\hline 9371.2583 & 4.203 & 9371.2584 & 5.331 & 9371.2584 & 6.265 \\
\hline 9372.2164 & 4.186 & 9372.2184 & 5.321 & 9372.2180 & 6.274 \\
\hline 9372.2356 & 4.202 & 9372.2188 & 5.330 & 9372.2188 & 6.255 \\
\hline 9373.2030 & 4.196 & 9373.2022 & 5.339 & & \\
\hline 9373.2186 & 4.194 & 9373.2186 & 5.336 & 9373.2190 & 6.267 \\
\hline 9373.2362 & 4.192 & 9373.2363 & 5.330 & & \\
\hline
\end{tabular}

$0.122 \AA$. The star $\beta$ Gem (K0 III) was used as comparison star.

On most nights, we obtained two spectra of the target, one before midnight and one after midnight. A total of 14 spectra with high $\mathrm{S} / \mathrm{N}$ ranging from 160 to 300 were obtained. The spectra obtained on Jan. 16 are noisy because of the bad weather. Information about the observations is given in Table 2, where JD of the observations corresponds to the midexposure and the phases were computed from the ephemeris of Fried et al. (1983).) Each spectrum was reduced according to the usual procedures: bias 


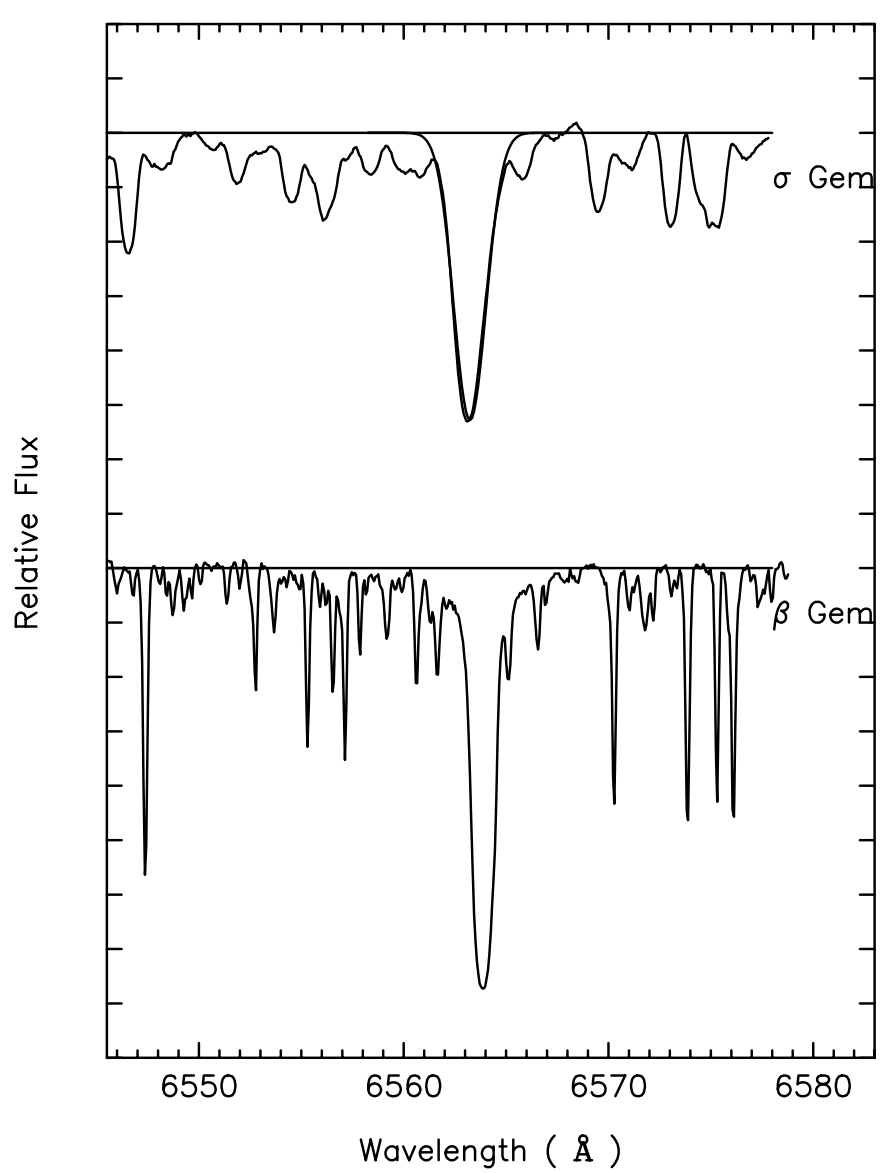

Fig. 2. Average spectrum of $\sigma$ Gem of the $\mathrm{H}_{\alpha}$ order after velocity shift corrections. The dotted curves illustrate the Gaussian fittings to the $\mathrm{H}_{\alpha}$ profiles

subtraction, dark subtraction, flat-field division, global slope correction and wavelength calibration. The precision of the calibration is better than $0.02 \AA$. The continuum level is established by choosing two line-free windows, approximately $2 \AA$ wide, centered at 6550 and $6578 \AA$. A straight line is then drawn through these two regions to define the continuum in each spectrum. Two representative spectra illustrating our continuum definition are shown in Fig. 2. The upper one is the average spectrum of $\sigma$ Gem and the lower one is of the comparison, $\beta \mathrm{Gem}$.

\section{The new light curves}

The new $U B V$ light curves (Fig. 1) are single-humped with asymmetry. The maximum brightness located around $\phi=0.2$ is found to be about $4.18 \mathrm{mag}$ in $V, 5.32 \mathrm{mag}$ in $B$ and $6.28 \mathrm{mag}$ in $U$, respectively. The minimum is located around $\phi=0.6$. The amplitudes of the light variation of $\sigma$ Gem in 1993/94 was about $0.1 \mathrm{mag}, 0.09 \mathrm{mag}$ and $0.07 \mathrm{mag}$ in $V, B$ and $U$ respectively. Comparing with the light curves of Olah et al. (1989) obtained in 1986, 1987 and 1988, we find that the distortion wave has evidently been migrating steadily.
Table 2. The spectroscopic observations of $\sigma$ Gem in 1993

\begin{tabular}{rcccc}
\hline & HJD & & & \\
No. & $2440000+$ & Date & Phase & $\begin{array}{c}\text { Exp. Time } \\
\text { (second) }\end{array}$ \\
\hline & & & & \\
1 & 9000.131 & 12.1 .1993 & 0.089 & 3000 \\
2 & 9001.075 & 13.1 .1993 & 0.137 & 2400 \\
3 & 9001.274 & 13.1 .1993 & 0.148 & 1800 \\
4 & 9002.076 & 14.1 .1993 & 0.189 & 1800 \\
5 & 9002.231 & 14.1 .1993 & 0.197 & 1800 \\
& & & & \\
6 & 9003.253 & 15.1 .1993 & 0.249 & 1800 \\
7 & 9003.306 & 15.1 .1993 & 0.252 & 1800 \\
8 & 9004.257 & 16.1 .1993 & 0.301 & 1500 \\
9 & 9004.346 & 16.1 .1993 & 0.306 & 2100 \\
10 & 9005.227 & 17.1 .1993 & 0.351 & 2100 \\
& & & & \\
11 & 9006.242 & 18.1 .1993 & 0.403 & 1800 \\
12 & 9006.340 & 18.1 .1993 & 0.409 & 1800 \\
13 & 9007.226 & 19.1 .1993 & 0.454 & 1200 \\
14 & 9007.294 & 19.1 .1993 & 0.458 & 1000 \\
\hline
\end{tabular}

The shape of the light curves implies large-scale spot activity on surface of the star. To study the spot activity furtherly from the light curves, a brief photometric analysis was carried out using the Wilson-Devinney program with spot approximation (Kang \& Wilson 1989; Zhai et al. 1994). The major Roche parameters are mainly adopted or estimated from the previous works on this system (Stawikowski \& Glebocki 1994; Eker 1986). With a two-spot model, the computed light curves give a good fitting to all the $V, B$ and $U$ light curves as shown in Fig. 1.

The result indicates that probably two cool spots exist on the primary component of $\sigma$ Gem in the season of $1993 / 94$. The one cooler and larger is located at the phase around $\phi=0.6$ and the other, smaller one is around the phase of $\phi=0.9$. Additional spots should also be present on the star to account for the general dimming from the unspotted level (4. 137 , Strassmeier et al. 1988) to our measured maximum light $\left(4^{\mathrm{m}} 18\right)$. Because of the considerable observational errors and the large gap between our two observational intervals, however, the resulting parameters of the spots are uncertain.

\section{4. $\mathrm{H}_{\alpha}$ variations}

The average spectrum of $\sigma$ Gemis shown in Fig. 2 which is obtained by adding together the 14 individual spectra after velocity shift corrections to the centers of $\mathrm{H}_{\alpha}$, agreeing to \pm 1 pixel. The center of $\mathrm{H}_{\alpha}$ is located by fitting a Gaussian profile as illustrated in Fig. 2. Subtracting the average spectrum from the shifted, individual spectra yields the residual spectra as shown in Fig. 3. Arrows 


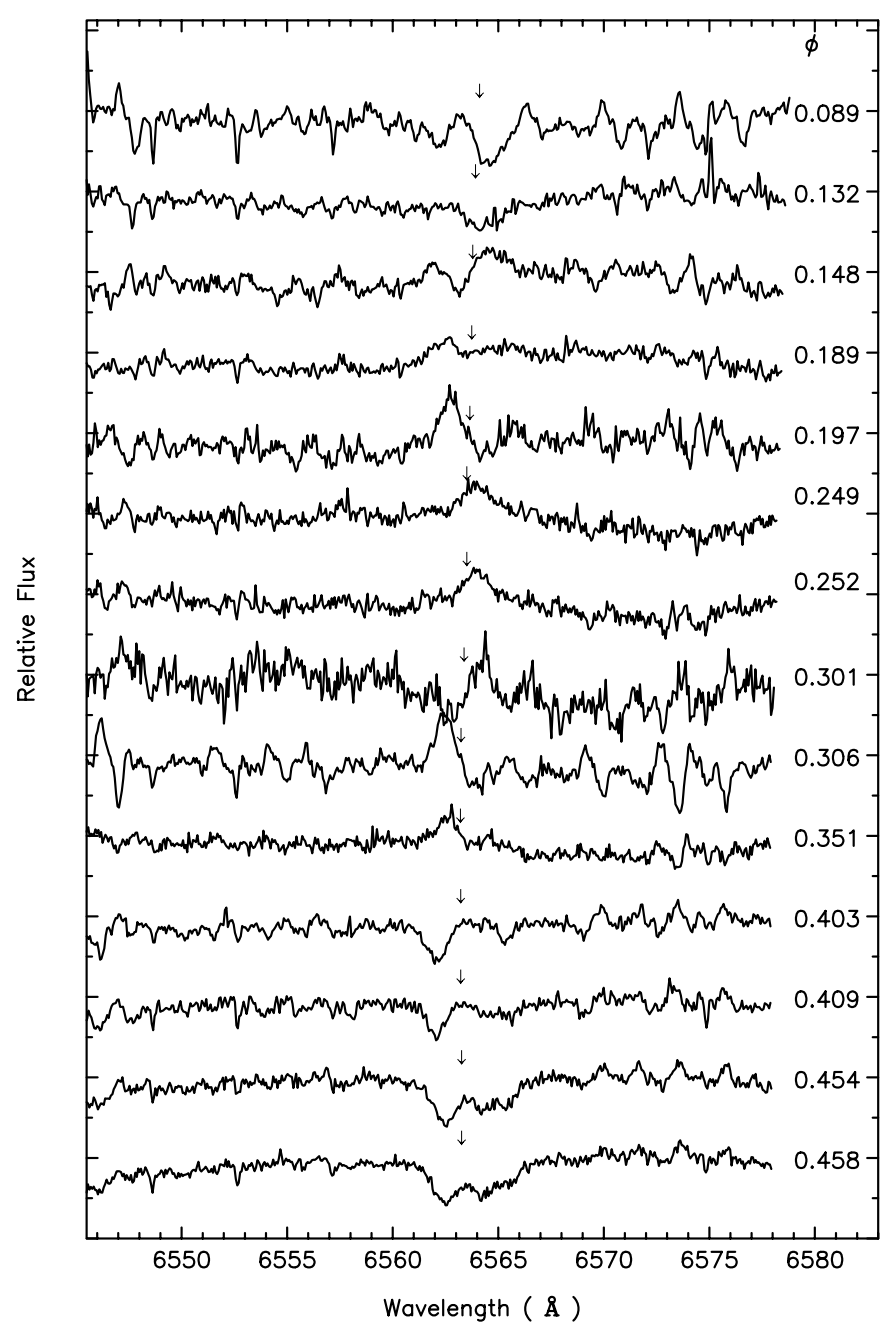

Fig. 3. Residual spectra obtained by abstracting the average spectrum from the individual spectra after velocity shift corrections. Phases are from Table 2. Arrows mark the centers of $\mathrm{H}_{\alpha}$

mark the centers of $\mathrm{H}_{\alpha}$. As can be seen in Fig. 3, the residual $\mathrm{H}_{\alpha}$ profiles show variations with phase. It is clearly in emission in scans 6 and 7 with phases around 0.25 , which corresponds to the maximum of the light curves. At phases with more spots in view (scans 1,2 and 13,14), the residual $\mathrm{H}_{\alpha}$ lines appear obviously in absorption.

Table 5 lists the values of the equivalent width (EW) and the central depth $\left(R_{\mathrm{c}}\right)$ of $\mathrm{H}_{\alpha}$ for each of the 14 individual spectra and the average spectrum of $\sigma$ Gem. These values are obtained by one Gaussian fitting. Figure 4 shows the variations of $\mathrm{EW}$ and $R_{\mathrm{c}}$ with phase according to Table 5. The dashed line represents the calculated $V$ light curve. It is obvious that the equivalent width and central depth of the $\mathrm{H}_{\alpha}$ profile show distinct anticorrelation with the light curve, (i.e., with the spot regions), though the spectroscopy covers only half of a period. The $\mathrm{H}_{\alpha}$ equivalent width shows the smallest value at maximum brightness, and tends to increase with the
Table 3. The variations of the $\mathrm{H}_{\alpha}$ profile of $\sigma$ Gem

\begin{tabular}{|c|c|c|c|}
\hline No. & Phase & $\begin{array}{c}\mathrm{EW}\left(\mathrm{H}_{\alpha}\right) \\
(\mathrm{mA})\end{array}$ & $R_{\mathrm{c}}$ \\
\hline 1 & 0.089 & 1060 & 0.459 \\
\hline 2 & 0.137 & 1005 & 0.469 \\
\hline 3 & 0.148 & 988 & 0.478 \\
\hline 4 & 0.189 & 995 & 0.479 \\
\hline 5 & 0.197 & 995 & 0.475 \\
\hline 6 & 0.249 & 966 & 0.502 \\
\hline 7 & 0.252 & 985 & 0.495 \\
\hline 8 & 0.301 & 985 & 0.495 \\
\hline 9 & 0.306 & 1007 & 0.481 \\
\hline 10 & 0.351 & 1002 & 0.493 \\
\hline 11 & 0.403 & 1078 & 0.473 \\
\hline 12 & 0.409 & 1082 & 0.475 \\
\hline 13 & 0.454 & 1125 & 0.455 \\
\hline 14 & 0.458 & 1136 & 0.452 \\
\hline Average & & 1042 & 0.47 \\
\hline
\end{tabular}

appearance of spots. It agrees well with the result from the analysis of the residual $\mathrm{H}_{\alpha}$ profiles above.

In general, for a temperature insensitive spectral line, the appearance of spots cause changes in the shape of the line profile, but not in its equivalent width (Vogt \& Penrod 1983). Thus, the variation of the $\mathrm{H}_{\alpha}$ equivalent width in $\sigma$ Gem is probably due to chromospheric $\mathrm{H}_{\alpha}$ emissions.

Many authors investigated the correlation between $\mathrm{H}_{\alpha}$ core emission and spot regions in RS CVn-type stars, and got different results. Weiler (1978) found a marginal correlation between $\mathrm{H}_{\alpha}$ emission and wave minimum for $\mathrm{RS}$ CVn, UX Ari and Z Her. A similar correlation holds for II Peg (Vogt 1981). In the Sun, the enhanced chromospheric emissions are from the plage regions visible in white light. If, according to the suggestion of Walter (1996), the solar analogy is valid, in case of $\sigma$ Gem there may be bright, spatially distinct plages where the enhanced $\mathrm{H}_{\alpha}$ core emission comes from at the phase of maximum light.

The average equivalent width of the $\mathrm{H}_{\alpha}$ absorption profile is measured to be $\mathrm{EW}=1042 \mathrm{m \AA}$ with an average central depth of $R_{\mathrm{c}}=0.47$ for $\sigma$ Gem. Strassmeier et al. (1986) gave a value of $\mathrm{EW}=972 \mathrm{m \AA}$ with $R_{\mathrm{c}}=0.51$ while Bopp et al. (1988) gave $\mathrm{EW} \simeq 1100 \mathrm{m \AA}$ and $R_{\mathrm{c}} \simeq 0.44$. The differences may be due to the variations of the active regions apart from the errors of measurements.

\section{Summary}

We made new $U B V$ photoelectric photometry and high resolution $\mathrm{H}_{\alpha}$ spectroscopy of the chromospheric active binary system $\sigma$ Gem in 1993-1994. The analysis of the light curves and the $\mathrm{H}_{\alpha}$ spectra yield the following results: 


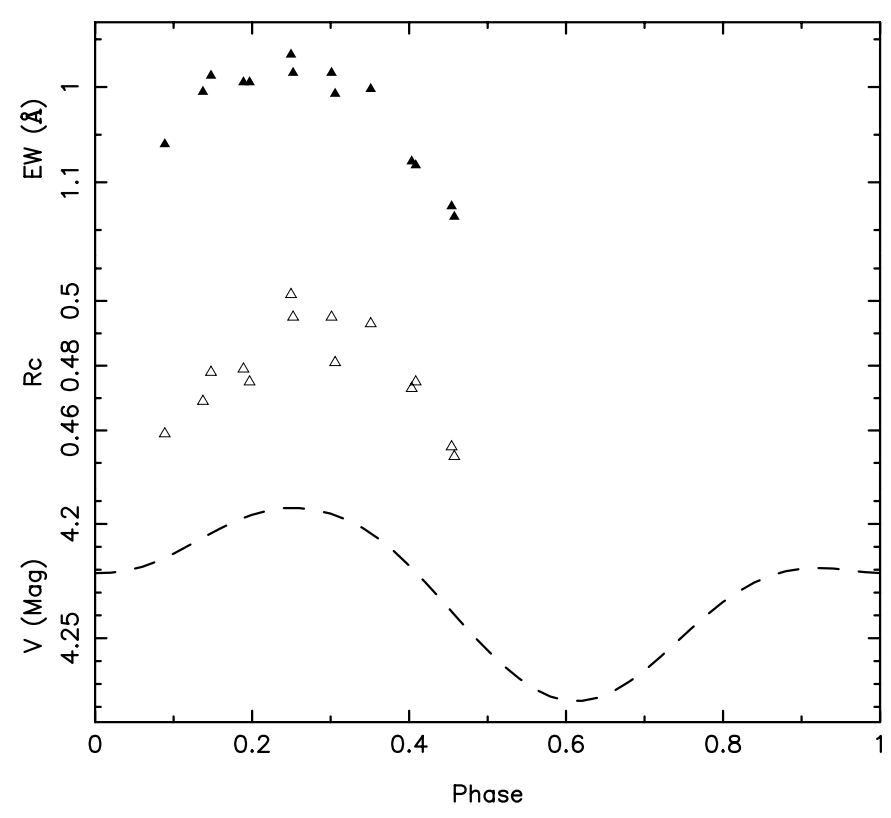

Fig. 4. The anti-correlations of the $\mathrm{H}_{\alpha}$ equivalent width (EW) and the central depth $R_{\mathrm{c}}$ with the distorted wave. The dotted line represents the $V$ light curve of $\sigma$ Gem

(1) The light curve migrated steadily and the distortion wave varied comparing to the previous observations, though the measurements show a considerable scatter. A brief photometric analysis, carried out using the WilsonDevinney method, suggests two cool spots on the primary component of $\sigma$ Gem around phases of 0.6 and 0.9 apart from additional spots causing the general dimming of the star.

(2) In connection with the photometric observation, the $\mathrm{H}_{\alpha}$ spetctroscopy of $\sigma$ Gem is analyzed. It is found that the core of the $\mathrm{H}_{\alpha}$ profile is varying with phase and time. The near to simultaneous photometry and spectroscopy enable us to find that the enhanced $\mathrm{H}_{\alpha}$ core emissions show distinct anti-correlation with spot regions. Our study suggests that there may be some plage-type regions around the maximum light, where the enhanced $\mathrm{H}_{\alpha}$ emission comes from.
Acknowledgements. This work is a contribution to the memory of our honoured Professor Zhai Disheng. He had been concerned with this work until his passing away. The authors would like to thank the Natural Science Foundation of China for financial support. Thanks are also due to Dr. J.X. Wang for his most helpful discussions.

\section{References}

Bopp B.W., Dempsey R.C., Manlak S., 1988, ApJS 68, 803

Bopp B.W., Dempsey R.C., 1989, PASP 101, 516

Cuby J.G., Bandrand J., Simon T., 1992, in: Proceedings of the 2nd MUSICOS Workshop, Catala C., Foing B.H. (eds.). Meudon Observatoire de Paris, p. 59

Eker Z., 1986, MNRAS 221, 947

Elgaray Z.Yi, Engvold O., Westergaard N.J., 1997, A\&A 318, 791

Frasca A., Catalano S., 1994, A\&A 284, 883

Fried R.E., Eaton J.A., Hall D.S., et al., 1983, Ap. Space Sci. 93, 305

Hall D.S., Henry G.W., Landis H.J., 1977, IBVS, No. 1328

Henry G.W., Eaton J.A., Hamer J., Hall D.S., 1995, ApJS 97, 513

Kang Y.W., Wilson R.E., 1989, AJ 97, 848

Olah K., Panov K.P., Pettersen B.R., Valtaoja E., Valtaoja L., 1989, A\&A 218, 192

Schrijver C.J., Mewe R., van den Oord G.H.J., Kaastra J.S., 1995, A\&A 302, 438

Smith S.E., Bopp B.W., 1982, Astrophys. Lett. 22, 127

Stawikowski A., Glebocki R., 1994, Acta Astron. 44, 393

Strassmeier K.G., Fekel F.C., 1990, A\&A 230, 398

Strassmeier K.G., Hall D.S., Eaton J.A., et al., 1988, A\&A 192, 135.

Strassmeier K.G., Weichinger S., Hanslmeier A., 1986, IBVS, No. 2937

Vogt S.S., 1981, ApJ 247, 975.

Vogt S.S., Penrod G.D., 1983, PASP 95, 565

Walter F.M., 1996, in: Stellar Surface Structure, IAU Symp., No. 176 , p. 355

Weiler E.J., 1978, MNRAS 182, 77

Zhai D.S., Foing B.H., Cutispoto G., et al., 1994, A\&A 282, 168 